\title{
The SCIENTIFIC REPRESENTATION OF TEMPORAL STRATIFICATION
}

\section{Maria Giulia Dondero}

University of Liège

\section{Introduction}

My study concerns the strategies of temporal stratification in astrophysics and archaeology's images. I plan to take into consideration a problematic issue which is transversal to contemporary scientific disciplines: the representation of the stratification of temporal layers. Whilst the disciplines chosen for this research, astrophysics and archaeology, are very different in terms of their subject of study, they do have a common point which is that a large number of their objects are invisible to the naked eye. More precisely, my objective is to compare the strategies of visual representation of the temporal layers stratified in these invisible objects.

\section{The Image and Visualisation in Astrophysics}

The two disciplines propose very different methods of research: showing the existence of an object and then studying its history by means of its physicochemical properties for astrophysics and showing the processes of constitution and conservation of objects in time for archaeology. But as a final objective of visual research, both aim for the representation of temporal strata, and more precisely seek to know how the present state of things prevents us from seeing the past in the case of archaeology and how the past and present of a star make it possible to predict the future in astrophysics.

What can be done in order to know the age, the moments of birth and death of the stars? It is through the collection of light according to the different wavelengths that the history of a star and the space-time depth of the sky can be identified. The different wavelengths measure the different sensibilities through which astral phenomena show themselves, give us information on the processes of formation, duration and explosion. Already, from detection frequencies we can form hypotheses about the duration of astral events, given that from the parts of the spectrum that are relevant we can predict the rhythms of development of these same events. 
Here I will take into consideration spectral analysis and the interconnection of detections allowing for prediction of the temporal evolution of the different stars. The characteristic of astrophysics is that the electromagnetic spectrum allows for easy transduction between one detection and another and one wavelength and another: each relevant wavelength can stand for a period of the life time of a star. Thanks to the electromagnetic spectrum, astrophysics is capable of easily making it possible to compare traces and data of various astral processes.

I'll begin with spectral analysis, by examining three types of images which show three ways to visually codify the temporal processes.

I'll start with the description of images-results, the final images of stars, which accumulate and integrate into their topology several different detections of light and which make up the iconography of what is called an "object", in this specific case, an astral object, characterized by a reconstruction of its history.

I will then go on to the second type of images, the "partial" visualizations of the stars which depend each on a single instrument of detection and which then have to be integrated with other visualizations in order to themselves become final images constituting an object.

The third type of images concerns visualizations of forces throughout different wavelengths (that I will call images-measure), which show themselves to be as maps which have yet to be configured into partial visualizations of a star.

The Images-result

Let's come back to the image of the constituted astral object, in this case the spirograph nebula (Photo 1) residues of gas ejected by a dying star.

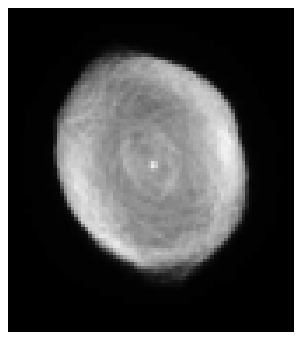

Photo 1. NASA et The Hubble Heritage Team. http:/ / apod.nasa.gov/apod/ap021214.html

These types of images, which are present especially in popularised scientific texts, gather the diverse captures of light obtained in the different wavelengths and purport to be documents identifying stars. This image is a mosaic constructed by integrated summation of the processes of transformation of the star which are codified into life phases. This image could be named as a temporal cartography that functions as a spa- 
tial synthesis of accumulated "photographs" and consequently identifies a precise object. This image codifies by means of eidetic, topological and chromatic configurations, information gathered by the different light detections. This final codification integrates the partial information and fixes it into an iconography to be diffused and to become a identifiable icon of the astral object. This "final" image can be understood as the translation and integration of several visualizations according to the different wavelengths which at last make possible an intelligibility of past transformations (and which can be useful to determine the future ones through the creation of stereotypes).

The integration of different partial visualizations produces a visual synthesis that consequently becomes a fictional stabilization and a temporary domestication of a potentially interminable observation and potentially infinite observations. It is this domestication which makes it possible to decide and stabilize the final form of an object and therefore the institutionalisation of this as an object of knowledge.

\section{Visualizations in Series}

Those we have called partial visualizations of a star show not the commensurability of detections and their final stabilization, but precisely different modes of detection of processes that are supposed to constitute a same astral object.

These four images show the residue of the supernova Cassiopeia $A$ (Photo 2).

Photo 2: http://chandra.harvard.edu/photo/1999/0237/. From left to right:
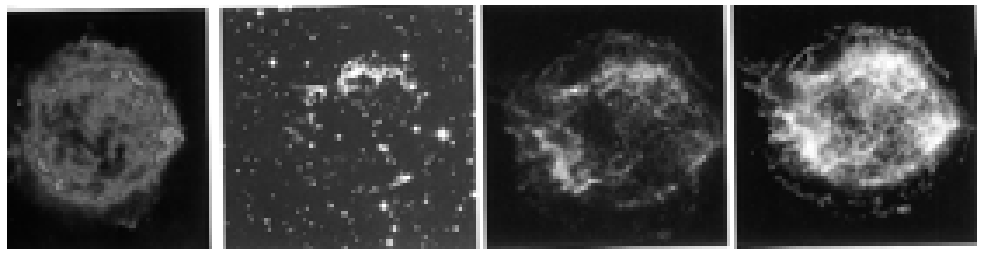

NRAO/AUI ; MDM/R. Fesen ; NASA/CXC/SAO ; NASA/CXC/SAO.

The first is a radio image, the second optic and the two last have been obtained by the Chandra telescope by means of $X$ rays at a distance of some years from each other. These images showing different moments of observation must be mutually commensurable in order to compose a temporal map which stratifies all the phases of the event (as we saw in the image of type 1). If we cannot identify the birth date or death of certain stars, for example, it is because we do not succeed in "optimising" the alignment of light traces obtained across the various image detections. Each phase of the life of a star, each process which 
happened in a certain time period, can only be captured by certain wavelengths. Some stars exist whose entire history we have been able to get, and others whose image-mapping still awaits confirmation by other detections that can be superimposed: very often we only succeed in dating the birth of a star a long time after its adult phase has been observed.

The image-mapping (type 1) will make up the synthesis of this series of partial visualizations. Every visualization is identifiable by similar values and by homogeneity of functioning.

The partial visualizations are indeed not images because they have not yet found an iconographic (iconic) ${ }^{1}$ or institutional (symbolic) stabilization (Dondero 2009). They are visualizations which, being distinct units, can be combined with other units according to some grammatical rules which have been constituted along the history of the discipline.

\section{Image-measures}

If we can consider the first image as an image mosaic which shows the summation of data by superimposition, and the four visualizations in series as mutual proofs of the existence of something that we will call an object, this third image (photo 3 ) is of a still different type.

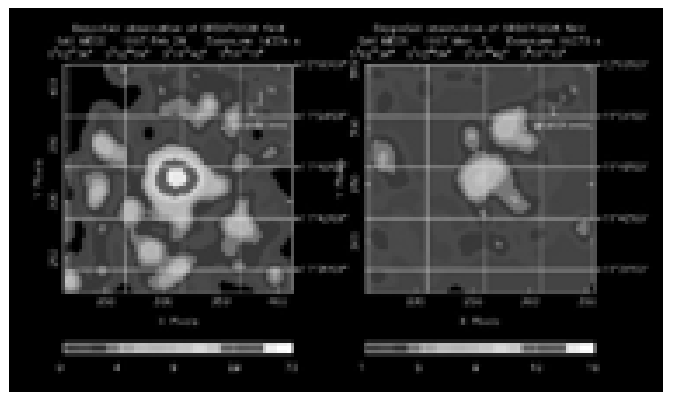

Photo 3: BeppoSAX/Team.

http: / / apod.nasa.gov/apod/image/9703/grb970228_sax_big.gif

This image picturing the first exact localisation of a burst of gamma rays by the satellite Beppo-SAX assumes the status of measure. We could say that it does not identify a burst of gamma rays as an object, but rather controlled reactions to the multiple attempts to identify the values relevant to the appearance of a phenomenon for which we had previously predicted a class of possible apparitions, appearances and func-

1 "I refer to the reformulation of the notion of peircian icon made by Bordron 2004" (Jean-François Bordron (2004) «L'iconicité », Ateliers de sémiotique visuelle, Hénault et Beyaert (dirs), Paris, P.U.F.) 
tions. This is not an image of something, but a map which makes it possible to visualize the different sensitivities of light in relation to a same event that has had different rhythms of making up. We thus obtain the values of this phenomenon through the visualization of the topological and chromatic differences and of the distances between them. These distances represent the space-time that is necessary so that the sources of the event cover all the domains of the sensibility of the spectrum, from gamma rays to radio waves. In fact, when we want to calculate the moment of explosion of a star, the image-measure shows us at what space-time distance relative to our space-time this explosion happened. We get this temporal distance through the calculation of the progressive difference of intensity of light emission in the different domains of wavelengths (different domains that in the image are represented by different colours).

\section{Autography and Allography in Astrophysics}

As we have said, the first type of image concerns a temporal cartography which implies an iconographic system which is accepted and stabilized: it concerns a final and isolated image, destined mostly for the popularisation books.

In the third case we are faced with cartography again, but this time it does not concerns a cartography that stabilizes an object through an iconography, but it rather pictures the topology of light values which will contribute to an understanding of the apparition of a phenomenon. It shows the border zones between the measuring which will constitute an event whose phases are determined again by the different sensitivities to light. With the first image, the image-result, we are at the end of the chain of codification of data, with the image-measure we are at the start of the chain.

If these two images are cartographies, it must be said that they show a very different connection with their instruments of fabrication. The image-measure reveals its enunciation devices which make it an image likely to become a place of operating and manipulating. The parameters that are shown allow the investigation to be followed, as much in terms of the manipulation of results as in terms of an eventual simulation which would make it possible to repeat the event investigated. This doesn't happen in the case of the image-result.

The image-result, on the other hand does not give us any spatial anchorage, no information about scales, or even about the space-time references of its enunciation (moreover the enunciations are multiple).

We can see that the procedures of translation of different detections in order to reach a commensurability and a stabilisation of the iconography of an object happened to the detriment of the revelation of the 
devices that have made possible the production and stabilisation of the object itself. In a certain way we could say that the final object, the object of knowledge, appears as soon as its enunciation has been concealed, hidden, almost eliminated-which is paradoxical. Moreover all the visualizations which present the devices that have made possible their enunciation are only intermediary images that function as carriers. In fact, in the second case, exemplified by the sequence of partial visualisations of Cassiopeia A, we are faced with visualizations in series that aim for the construction of a visual repertory, a grammar that constitutes a virtual environment which can be useful to model other astral realities to come.

These visualizations no longer concern images in the classical sense of the term, that is images constituted as such through the stabilization into an iconography. These visualizations can build a grammatical (virtual and modelling) environment of the possible. The data of each visualisation therefore becomes available for the actualization of this virtual environment (that is modelled by inscribed data and modelling data to come).

Indeed, even if astrophysics aims firstly to take account of an object of analysis (an object in itself), it aims secondly to make this object easily manipulated, in view of the production of hypotheses which can make it possible to reuse the data incorporated into this object for future research projects (this is why it is necessary to create a system of notation to then allow for a the diagrammatical transposition of results).

With regard to notation, I would like to propose a clarification in relation to two concepts which are of great interest to me and which were postulated by the American philosopher Nelson Goodman. If the system that Goodman (1968) considers autographic concerns the fixing of the density of traces of a process onto a supporting medium, the system of allography on the other hand concerns strategies for digitalizing of traces and data in order to construct a system of modelling of the latter via a system of notation (where the elements are disjointed and recombinable like the letters of an alphabet).

We could affirm that the autographic image of type 3 is proof of a unique phenomenon that is observed or detected which can not be repeated (this is why the support with the inscription keeps its relevance), while the allographic system of visualisations does not make relevant the anchorage of data on a support and allows the reproducibility and the modelling. It offers the possibility to scientists to pursue a course of simplification/schematisation which would make it into a sort of image-model, usable for other phenomena to be investigated.

The visualisations in the system of allography have the status of texts of instructions: these visualisations are used to accomplish other investigations; they are predictive images oriented toward the future. 
A diagram could be described as a device that is anchored in data (autography) and at the same time is transposable, to other data (allography) through a system of notation, in order to became un instrument of modelling and a device for future figurative imagination (see Stjernfelt 2007).

\section{The Superimposition of Images in Archaeology}

At present, I will briefly deal with the strategies of image production in archaeology and notably geophysical prospecting, non-invasive methods, ${ }^{2}$ making possible the detection of processes which have governed the formation of the subsoil. I will not focus on invasive methods such as excavation, which are methods that have become more and more illegitimate. In fact, excavation, where the measurements obtained can not be entirely repeated and verified, voids the principle of the scientific image constructed in a laboratory-a principle which is the equivalent of the image in astrophysics and of all the disciplines whose methods of inquiry are deemed as verifiable and subject to falsification. I will take into consideration the aspect of archaeology which is based on geophysical prospecting that exploits physical properties-as for example, electrical resistance-, to take samples of information on material structures of the subsoil and which allow for total verification of the results of the investigation. These methods make it possible to construct gen-

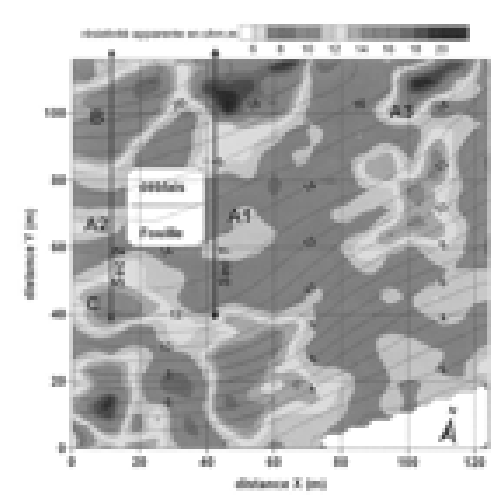
uine maps representing the distribution of physical properties in the different zones of the subsoils.

The following image (photo 4), which appears, in terms of figurative syntax, ${ }^{3}$ as very similar to the third image described in the domain of astrophysics, belongs to electrical prospecting.

Photo 4. Bordeneuve in Beaugas, Lot-etGaronne, 1988. Superposition of an horizontal electric image and a topography. Image published in Martinaud (2005).

2 It concerns non-invasive procedures by inventories which are connected to a relative chronology that aims to study the relation between strata of the soil and subsoil, which is the opposite of invasive excavation which is linked to an absolute chronology that aims to make objects and events correspond with precise phases from the past.

3 By figurative syntax, I mean the way forms are inscribed and become stable on a support of inscription. See Fontanille (2004). 
This method makes use of a physical property, electrical resistance, to take information samples of the structure of the soil. This physical property makes it possible to characterise the difficulty that the electrical current meets while passing through certain materials, like for example, clayey soils.

Archaeology, like astrophysics' visual system, works by the superimposition of images: the most interesting superimpositions place the images-measurements of electric and electromagnetic prospecting in relation with images produced by aerial prospecting (which brings us back to type 3 of our classification). When we superimpose the images produced by aerial prospecting (in brown in the photo), and those of electromagnetic prospecting (in grey-green in the photo), we obtain images such as this one (photo 5) where the diversity of the methods of detection, in contrast with astrophysics, produces an irreducible heterogeneity. This heterogeneity offers what we might call a diagrammatic intravision.

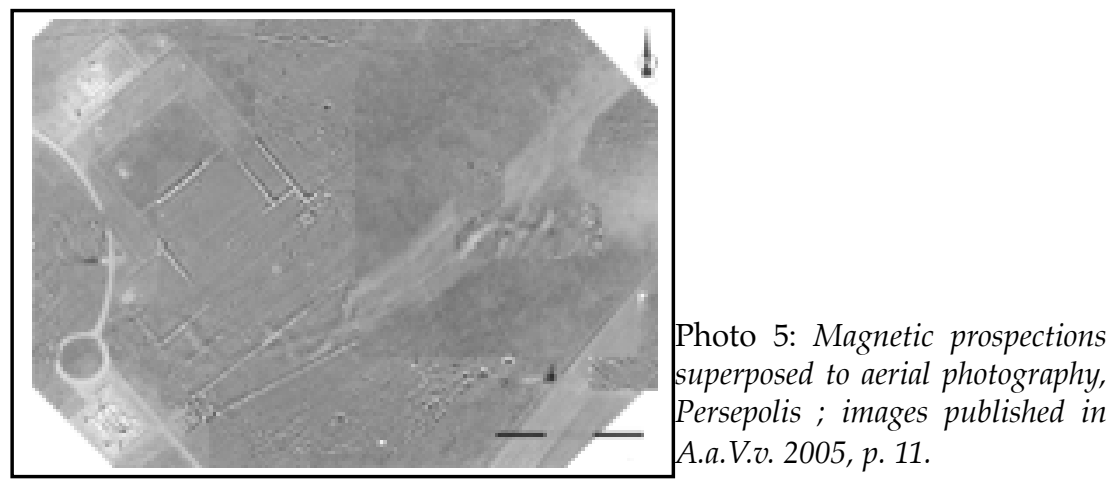

In this image we are confronted by two juxtaposed figurative syntaxes: diagrammatic intravision is, in fact, a chink between two systems of relevance, between two regimes of research. Diagrammatic intravision is a form of new vision produced by reciprocal transposition of two regimes of perceptive relevance. Diagrammatic intravision is an inbetween vision which allows us to see a possible future transposition of relations between two systems of research in order to accomplish an action of manipulation.

We can clearly see that archaeology is a different process to astrophysics: we could say that here we have partial visualisations as well, but these partial visualisations do not make it automatically possible to transpose totally, nor does it guarantee homogeneity which, on the contrary, is guaranteed by the electromagnetic spectrum in astrophysics. Here transposability, in order to be operational, must remain merely potential, possible. In this last image this possible commensurability is 
supported in terms of expression by networks of relations which are characterised in contrasting ways. But it is not just about simple isomorphism between the plans. Diagrammatic intravision is dynamic by way of contrast because it makes it possible to reconstruct patterns that are evolving, or even understand syntaxes, both in terms of the plane of the enunciate (process of events observed) and the plane of enunciation (process of research).

Through these methods of research we do not have definitive objects as a result, the object-time here can not necessarily be reconstructed through an iconography of the object, as it can be in astrophysics with the astral object. In archaeology as final images we only get maps which can give us indications of future actions which are more or less possible and promising.

\section{Integrated Superimposition and Diagrammatic Superimposition}

The images-measurements share the same figurative syntax in astronomy and in archaeology, but the final composite images are quite different and it is on this that I would like to focus my attention to reach conclusions.

The images in astrophysics aim, in short, to summon, superimpose, integrate -swallow up almost- the traces, to reduce the heterogeneity of light captures in a single image as for example in the cluster of galaxies shown hereinafter (photo 6) which shows very clearly how the final image integrates the images coming from different detections in order to stabilise the identity of an object.

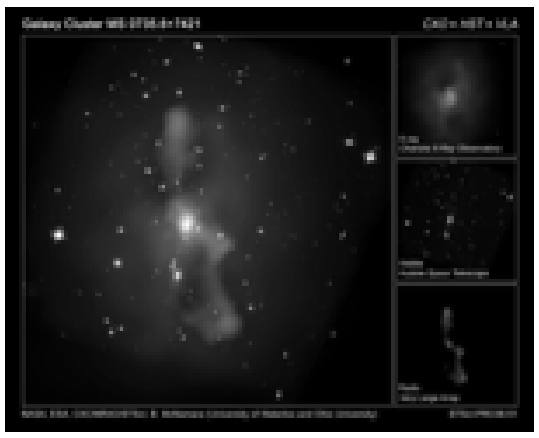

Photo 6 :

http:/ / imgsrc.hubblesite.org/hu/db/ 2006/51/images/a/formats/print.jpg

When conceived in this way, the image of a star has a double objective: first to construct a good reference for the star in question (autography), and second the creation of a grammatical basis for comparison with other stars by virtue of composing and recomposing the relations between temporal processes and light collects. These connections supply commensurable and superimposable modules (allography). In 
astrophysics the "correct" reference is reached when the manipulations it allows through partial visualisations contribute to the formulation of new hypotheses and discoveries. This happens when its modules can be "articulated" and the different systems of detection can be integrated. In this final image of the cluster of galaxies everything happens as if the visualisations that make it up possessed a "transparent" consistence with regard to other visualisations; this transparency, or even commensurability, is guaranteed by the rules of notationality, the end objective being a superimposition which homogenises the traces of different detections. Everything happens as if the partial visualisations aimed to compact the final image which is dispersed in the universe -because it involves constructing simulations of cohesion of data which in reality are diffused in time and space. The final image has assimilated all the measures: that is to say that the arrival point of the astronomical iconography is the homogenisation of traces. This image has the objective of compacting the different processes of the universe into a cartography which totalises and integrates the different temporal processes by transforming them into phases: the birth growth and death of a star.

The images in astrophysics aim to transform the processes in phases.

And yet, all of this is far from being true for archaeological images where a measure and method of detection is never integrated or integratable with the other. In archaeology the images-cartographies no longer aim for the integration of traces, nor to become model images for a series of other investigations; quite on the contrary, they represent the heterogeneity of research methods (diagrammatic intravision), their impossibility to be recomposed into a unit. This impossibility of homogenisation allows not only for a double or multiple vision of the same event, but also and in particular, a metavision of the possible commensurabilities of points of view. It is the non-commensurability which allows the research in the case of archaeology, a research which is based on the differentiation of traces. Each composite image in archaeology must show the diversity of the methods of interconnectivity of the different parts of the subsoil; each stratified method of investigation in the composite image pictures a process of exfoliation of something compact. The visual set of results obtained through the different parameters show the effort to extract the heterogonous where there is no apparent homogeneity.

All in all, the function of the images in archaeology is quite different to that which they assume in astrophysics: from the homogenous and the compact, it is necessary to achieve an exfoliation of the subsoil, by showing all of its diversity. Each layer must show another, or several others, from which it can be extracted: it is by dissimilation that we can succeed in seeing and operating. It is the same process of extraction that the image is supposed to show by means of diagrammatic intravision. 


\section{To conclude}

The images in astrophysics and archaeology present two different types of virtuality of the object of perception: the object in astronomy is hidden, because it is too distant and diffuse, while the archaeological object is hidden because its stratification is too compact. It follows that in astrophysics the images update the object by assimilations that compact the evanescent, through commensurable light models of the spectrum while in archaeology the images function by differentiation of what they actualise, that is to say by dissimilation within a stratified and compact matter. In astrophysics, we operate by superimposition and adjustment of temporal modules and stocks of light; in archaeology, by differentiation of thin layers. All this creates a different relation between dating, which in astrophysics is based on a recording of processes: we obtain a dating by translating processes into phases. In archaeology it is the opposite: it is by exfoliation of the layers in depth and thus phases of a territory that we can reconstruct processes of occultation and formation of residues.

\section{References}

BORDRON, Jean-François.

2004. "L'iconicité », Ateliers de sémiotique visuelle, Hénault et Beyaert (dirs), Paris, P.U.F.

DONDERO, Maria Giulia.

2009. "L'image scientifique : de la visualisation à la mathématisation et retour", Nouveaux Actes Sémiotiques, [ en ligne ]. Recherches sémiotiques. http: / / revues.unilim.fr/nas/ document.php?id=2907.

GOODMAN, Nelson.

1968. Languages of Art (London, Bobbs Merrill).

MARTINAUD, Michel.

2005. "Prospection géophysique et sites préhistoriques", A.a. V.v. Dossiers d'Archéologie, 308, 30.

STJERNFELT, Frederik.

2007. "Diagrammatology", An Investigation on the Borderlines of Phenomenology, Ontology, and Semiotics, series vol. 336 (Springer Netherlands: Synthesis Library). 